\title{
Analysis of Systemic and Cutaneous Inflammatory Immune Response in Canine Atopic Dermatitis
}

\author{
Tiago Cunha Ferreira', Juliana Furtado Lima-Verde', José Alexandre da Silva Júnior', \\ Társsila Mara Vieira Ferreira', Daniel de Araújo Viana² \& Diana Célia Sousa Nunes-Pinheiro'1
}

\begin{abstract}
Background: Canine atopic dermatitis (CAD) is a chronic and inflammatory disease present in veterinary dermatological practice. The inflammation in CAD is triggered by environmental allergens and skin microorganisms, which are responsible for the worsening of cutaneous lesions. This continuous activation of skin inflammatory process can induce the production of free radicals that also contribute to cellular damage and ultimately leads to changes in blood parameters in dogs with CAD. Although there are reports of inflammatory parameters in CAD, there are a lack of studies correlating skin lesions, blood leukocytes and oxidative stress. Based on that, this study aimed to evaluate the integumentary and systemic inflammatory response in dogs with atopic dermatitis.

Materials, Methods \& Results: Dogs with confirmed diagnosis of canine atopic dermatitis $(\mathrm{n}=10)$ were divided in two groups according to CADESI-IV: AI, with CADESI between 0-10, AII, with CADESI between 10-34, and control group (n $=5$ ). Blood-biochemical and histological analysis were performed to access systemic and cutaneous inflammatory response. AII group tended to higher neutrophil and eosinophil counts, as well as neutrophil/lymphocyte ratio (NLR) when compared to AI. The albumin was lower in AII compared to AI and control $(P<0.05)$, while total bilirubin and malondialdehyde (MDA) did not differ between groups. NLR $(\mathrm{r}=0.64$ and $P=0.04)$ and MDA $(\mathrm{r}=0.54$ and $P=0.1)$ were positively correlated with CADESI, while albumin was negatively correlated with CADESI $(\mathrm{r}=-0.79$ and $P=0.005)$. Histopathological analysis revealed a larger number of neutrophils, macrophages and mast cells in AI and AII than in control group $(P<0.05)$. Discussion: In this study it was possible to evaluate the systemic and cutaneous leukocyte dynamics in CAD. Skin inflammation induces the production of chemotactic molecules contribute to neutrophil outflow from blood vessel toward the affected tissue, which can be visualized as perivascular inflammatory infiltrate and exocytosis. At systemic level, there was a tendency to increase in total leukocytes and circulating neutrophil count in group AII, as well as in neutrophil/lymphocyte ratio (NLR), when compared to AI. The NLR is a widely available and inexpensive laboratory biomarker that quantifies systemic inflammation, being used in human medicine to evaluate prognosis in different types of cancer. In our study, dogs in AII group showed an increased NLR compared to AI and control, which demonstrates the influence of skin injury in systemic parameters. Furthermore, AII group is composed of dogs with greater lesion state, which reflects in higher NLR values. Since this disease is known by its chronicity and may remain stable for years, NLR may be a novel biomarker to evaluate acute exacerbation in $\mathrm{CAD}$ and could potentially explain why some patients have longer crisis duration and frequent flares. The maintenance of the inflammatory state also induces the production of oxidizing substances, which possibly exceed the total antioxidant capacity, generating a situation of oxidative stress, which can result in damage to membrane lipids and release of their products. MDA is reliable and is the most commonly used marker of the overall lipid peroxidation level and the presence of oxidative stress. This result may be related with the antioxidant system, such as albumin and bilirubin, which was able to promote an efficient control of oxidant substances. In conclusion, the presented data demonstrated an inflammatory process progression as well as introduced NLR as a potential marker of disease exacerbation in CAD.
\end{abstract}

Keywords: systemic inflammation, mast cells, allergic skin disease, NLR. 


\section{INTRODUCTION}

Canine atopic dermatitis (CAD) is a chronic, pruritic and inflammatory skin disease that has a multifactorial etiology and may be mainly associated with genetic predisposition, changes in epidermal barrier and microbial imbalance [7,30]. These factors activate the immune system, triggering an inflammatory process that culminates in tissue damage and ultimately leads to systemic changes [34,35]. Pruritus is the main clinical manifestation of CAD, but erythema, alopecia, hyperpigmentation and lichenification can also be present $[10,11]$.

The inflammatory process in CAD contributes to altering and reducing the diversity of the skin microbiota, which facilitates the establishment of recurrent skin infections [26]. Bacterial and fungal overgrowth, known as dysbiosis, are the most common observed manifestations, mainly by Staphylococcus pseudintermedius and Malassezia spp. [6,13] and are associated with exacerbation and maintenance of inflammatory process [28].

The activation of inflammatory cells induces the production of free radicals, which may oxidate cellular membrane and potentially contributes to immunopathogenesis of CAD $[2,5,15]$. To control the oxidative skin damage, enzymatic and nonenzymatic antioxidant molecules are activated in order to improve the defense systems and neutralize free radicals [21].

To assess tissue damage in CAD, a clinical measurement of the integumentary inflammatory process was proposed, based on the analysis of lesion patterns [25]. In this sense, the aim of the present study was to evaluate the tissue and systemic inflammatory response according to skin lesion grade of dogs with atopic dermatitis.

\section{MATERIALS AND METHODS}

\section{Animal sampling}

Fifteen dogs of different breeds from the Metropolitan Region of Fortaleza, ranging in age from 01 to 08 years-old, were included in the study. The dogs in experimental group $(n=10)$ have history and clinical signs compatible with CAD [11] in addition to being submitted to exclusion of other pruritic dermatopathies through skin scrapping, fungal culture and elimination diet. The control group $(n=5)$ was consisted of dogs from Ceara Fire Department, and presented clinical and pathological evaluation within normal range for the species. All dogs were seronegative for Leishmaniasis and had no use of immunosuppressive drugs for two weeks prior to biopsy.
The experimental group was further clinically evaluated according to the criteria established by the International Committee on Allergic Diseases of Animals (ICADA). These criteria use lesion parameters at specific anatomical sites (Canine Atopic Dermatitis Severity Index 4 - CADESI IV), allowing the classification of the disease in: CAD in remission: up to 10 ; Mild CAD: between 10-34; Moderate CAD: between 35-59; Severe CAD: between 60-180 [25].

\section{Blood sampling and hemato-biochemical analysis}

Blood samples were collected by jugular venipuncture and stored in EDTA and non-anticoagulant tubes for evaluation of systemic parameters. The following parameters were evaluated: total leukocytes, neutrophils, lymphocytes, eosinophils, neutrophil/ lymphocyte ratio and biochemical parameters as serum albumin, total bilirubin, alanine aminotransferase (ALT), creatinine and malondialdehyde (MDA).

To evaluate lipid peroxidation, MDA analysis was performed, where $250 \mu \mathrm{L}$ of serum was added to $400 \mu \mathrm{L}$ of perchloric $\operatorname{acid}^{1}(35 \%)$ and taken to water bath $\left(37^{\circ} \mathrm{C}, 01 \mathrm{~h}\right)$. The mixture was centrifuged $(1400$ $g ; 10 \mathrm{~min}$ ) and $600 \mu \mathrm{L}$ of the supernatant was added to $200 \mu \mathrm{L}$ of $1.2 \%$ thiobarbituric acid ${ }^{2}$. This mixture was taken to water bath $\left(95^{\circ} \mathrm{C}, 30 \mathrm{~min}\right)$. After cooling, the reading was performed in a spectrophotometer (535 $\mathrm{nm})$. The results obtained were expressed as nmol/ $\mathrm{mL}$ [9].

\section{Tissue sampling}

For histopathological analysis, skin biopsies were collected from ventral areas with inflammatory lesions using a $5 \mathrm{~mm}$ punch. Papules and pustules, if present, were avoided. Skin biopsy of control animals was also performed from the same regions. The samples were fixed in $10 \%$ buffered formalin and further processed by conventional histological techniques. Histological sections were stained with Hematoxylin and $\operatorname{Eosin}^{1}(\mathrm{H} \& \mathrm{E})$ for analysis of histological changes and Toluidine Blue ${ }^{1}$ for mast cell evaluation. Qualitative evaluation of morphological and morphometric parameters was performed and these parameters were classified as present or absent. For inflammatory cells evaluation, a quantitative analysis was performed in 10 different fields. The analyzes were performed under light microscope with magnification of $200 \mathrm{x}$ and $400 \mathrm{x}$, according to the average subjective perception of two observers. 


\section{Statistical analysis}

Statistical analysis was performed with the aid of Graph Pad® ${ }^{\circledR}$ software ${ }^{3}$. Student's t-test was used to evaluate differences in values determined between control and atopic groups. The correlation between CADESI and NLR, Albumin and MDA parameters were evaluated with Pearson's correlation test. In all cases, differences were considered significant when $P<0.05$.

\section{RESULTS}

\section{Clinical parameters}

As previously reported, the clinical evaluation of dogs was based on CADESI-IV, which provides lesion scores according to the following alterations: erythema, lichenification and excoriation/alopecia. Subsequently, dogs with atopic dermatitis were divided into two groups: Atopy I (AI), dogs with CADESI between 0-10, and Atopy II (AII), dogs with CADESI between 10-34. The third group was composed of control dogs, which had no clinical or hemato-biochemical abnormalities (C).

\section{Systemic inflammatory parameters}

The evaluation of systemic inflammation was based on parameters of leukogram, albumin and MDA (Table 1). There was an increase in neutrophil and eosinophil count as well as in neutrophil/lymphocyte ratio (NLR) in group AII when compared to control group. There was no difference in total leukocytes, neutrophils, NLR, and eosinophils between AI and AII, although an increasing tendency was observed in AII. A moderate positive and significant correlation between NLR and CADESI was observed ( $\mathrm{r}=0.64$ and $P=0.04$ )

Regarding biochemical evaluations (Table 1), the AII group presented lower albumin concentration than control and AI groups, while Total Bilirubin and serum MDA levels did not vary between groups. A strong negative and significant correlation between Albumin and CADESI was observed $(\mathrm{r}=-0.79$ and $P=0.005)$, while moderate positive correlation between MDA and CADESI was observed, although this correlation was not statistically significant $(\mathrm{r}=0.54$ and $P=0.1)$.

\section{Cutaneous inflammatory parameters}

Epidermal alterations and dermal inflammatory infiltrate were observed in groups AI and AII, whose data are provided in Table 2. Atopic animals presented acanthosis, hydropic degeneration, spongiosis, exocytosis and orthokeratosis in epidermal layer (Figure 1B and 1C).
Regarding dermal perivascular inflammatory infiltrate, it was characterized by neutrophils, eosinophils, lymphocytes, macrophages, and mast cells (Figure 1E, 1F, 1H, and 1I). The quantification of these cells was also performed, and an increase of neutrophils, mast cells and macrophages were observed infiltrating the dermis of atopic dogs when compared to control group $(P<0.05)$ [Table 2]. The difference between groups AI and AII, however, was not observed.

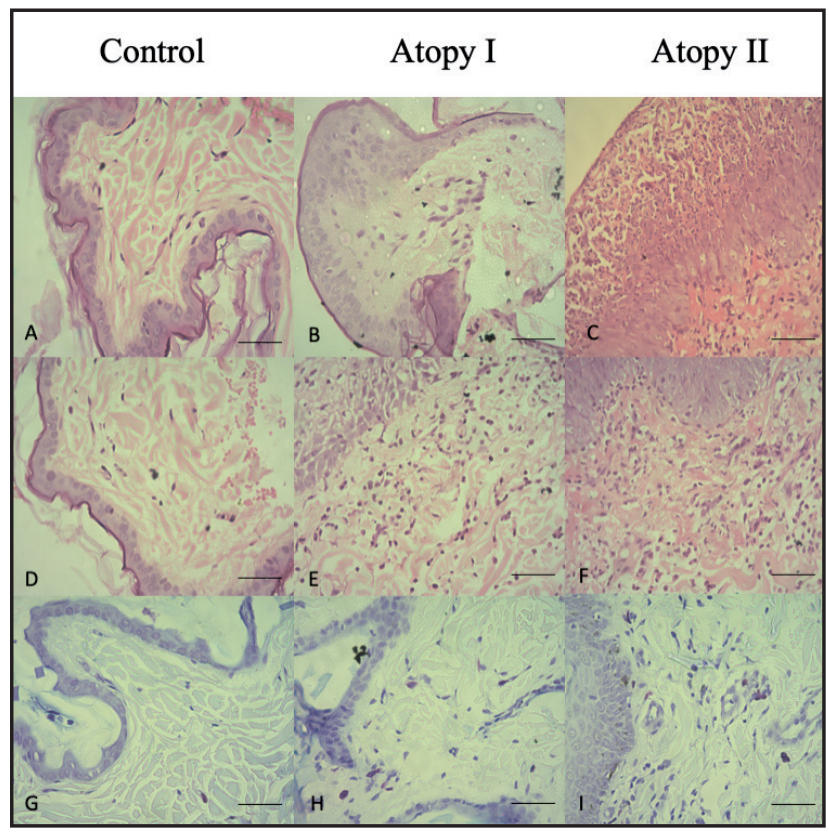

Figure 1. Histopathological aspects of dogs with CAD. A \& D- Represent the control group (H\&E), which shows no visible dermal and epidermal changes. B- It is possible to observe the epidermal layer thickening $(\mathrm{H} \& \mathrm{E})$. C- There is epidermal thickening, hydropic degeneration of keratinocytes, as well as neutrophilic exocytosis (H\&E). E \& F- Highlight the presence of inflammatory infiltrate in dermis of atopic dogs (H\&E). H \& I- It is possible to observe mast cells composing the dermal inflammatory cells of atopic dogs. G- Represents the control group, with a reduced number of these cells (Toluidine Blue). [Scale bar= $1 \mu \mathrm{m}$ ]. Original magnification: 400x.

\section{DISCUSSION}

Canine atopic dermatitis is an inflammatory skin disease that involves a complex immunopathological mechanism [30]. This disease presents clinically in different ways, requiring an individual approach according to lesion degree. In the present study, the leukocyte analysis was performed to assess inflammatory response, while albumin, total bilirubin and MDA evaluation aimed to estimate oxidative disturbs. Although other studies have already been performed correlating CADESI with blood parameters and lymphocytic phenotype in CAD [22], no clinical research was previously performed correlating blood and tissue inflammatory response in this disease according to CADESI. 
Table 1. Hematological, biochemical and clinical assessment parameters in control and atopic dogs.

\begin{tabular}{cccc}
\hline Hemato-Biochemical Parameter & Control & Atopy I (AI) & Atopy II (AII) \\
\hline Total Leukocytes $\left(10^{3} / \mu \mathrm{L}\right)$ & $10.20 \pm 1.53$ & $8.52 \pm 0.82$ & $11.58 \pm 2.01$ \\
Neutrophils $\left(10^{3} / \mu \mathrm{L}\right)$ & $6.38 \pm 0.96^{\mathrm{a}}$ & $6.39 \pm 0.89^{\mathrm{ab}}$ & $8.21 \pm 1.99^{\mathrm{b}}$ \\
Lymphocytes $\left(10^{3} / \mu \mathrm{L}\right)$ & $1.98 \pm 0.81$ & $1.25 \pm 0.16$ & $1.57 \pm 0.83$ \\
Neu/Lym Ratio & $3.77 \pm 1.3^{\mathrm{a}}$ & $5.18 \pm 1.1^{\mathrm{ab}}$ & $6.27 \pm 1.8^{\mathrm{b}}$ \\
Eosinophils $(\mathrm{cell} / \mathrm{s} / \mathrm{L})$ & $102 \pm 98.4^{\mathrm{a}}$ & $272.2 \pm 126.9^{\mathrm{ab}}$ & $466.2 \pm 216.1^{\mathrm{b}}$ \\
Albumin $(\mathrm{g} / \mathrm{dL})$ & $3.49 \pm 0.1^{\mathrm{a}}$ & $3.54 \pm 0.3^{\mathrm{a}}$ & $2.78 \pm 0.3^{\mathrm{b}}$ \\
Creatinine $(\mathrm{mg} / \mathrm{dL})$ & $0.9 \pm 0.3$ & $1.1 \pm 0.1$ & $1.2 \pm 0.1$ \\
ALT $(\mathrm{U} / \mathrm{L})$ & $58.1 \pm 20.3$ & $44.7 \pm 30.2$ & $60.4 \pm 25.1$ \\
Total Bilirubin & $0.4 \pm 0.2$ & $0.6 \pm 0.5$ & $0.5 \pm 0.4$ \\
Total Protein $(\mathrm{g} / \mathrm{dL})$ & $7.04 \pm 0.2$ & $7.94 \pm 0.4$ & $7.12 \pm 0.9$ \\
MDA (nmol/mL) & $0.86 \pm 0.09$ & $0.87 \pm 0.08$ & $0.91 \pm 0.15$ \\
CADESI-IV & & $7.6 \pm 1.5^{\mathrm{a}}$ & $29.6 \pm 10.8^{\mathrm{b}}$ \\
\hline
\end{tabular}

Results are expressed as the mean \pm standard deviation. Different letters show statistically significant differences $(P \leq 0.05)$ among control and atopic groups.

Table 2. Histopathological changes in control and atopic dogs.

\begin{tabular}{cccc}
\hline Epidermal Alteration & Control & Atopy I $(\mathrm{n}=5)$ & Atopy II $(\mathrm{n}=5)$ \\
\hline Acanthosis & Absent & Present $(\mathrm{n}=2)$ & Present $(\mathrm{n}=3)$ \\
Hydropic Degeneration & Absent & Present $(\mathrm{n}=2)$ & Present $(\mathrm{n}=2)$ \\
Spongiosis & Absent & Present $(\mathrm{n}=2)$ & Present $(\mathrm{n}=2)$ \\
Exocytosis & Absent & Absent & Present $(\mathrm{n}=1)$ \\
Parakeratosis & Absent & Absent & Absent \\
Orthokeratosis & Absent & Present $(\mathrm{n}=2)$ & Present $(\mathrm{n}=1)$ \\
\hline Inflammatory Cells in Dermis & & & $25.4 \pm 14.7^{\mathrm{b}}$ \\
Neutrophils & 0 & $11.3 \pm 8.12^{\mathrm{ab}}$ & $9.2 \pm 5.6$ \\
Eosinophils & 0 & $7.8 \pm 3.4$ & $14.7 \pm 6.9$ \\
Lymphocytes & 0 & $8.4 \pm 3.3$ & 0 \\
Plasma Cells & 0 & 0 & $33.6 \pm 10.1^{\mathrm{b}}$ \\
Macrophages & $11.2 \pm 7.4^{\mathrm{a}}$ & $21.3 \pm 11.7^{\text {ab }}$ & $19.8 \pm 11.8^{\mathrm{b}}$ \\
\hline Mast Cells & $6.1 \pm 0.89^{\mathrm{a}}$ & $18.4 \pm 7.86^{\mathrm{b}}$ & $\mathrm{y}$
\end{tabular}

Results are expressed as number of affected dogs/total of dogs for epidermal changes. Neutrophils, eosinophils, lymphocytes, plasma cells, macrophages and mast cells are expressed as mean \pm standard deviation. Different letters show statistically significant differences $(P \leq 0.05)$ among control and atopic groups.

In this study, it was possible to evaluate the systemic and cutaneous leukocyte dynamics in CAD. At systemic level, there was a tendency to increase in total leukocytes and circulating neutrophil count in group AII, as well as in neutrophil/lymphocyte ratio (NLR), when compared to AI. At tissue level, it was observed a neutrophil accumulation in perivascular inflammatory infiltrate, and sometimes the migration of these cells towards the epidermis (Figure 1C).
Skin inflammation induces the production of chemotactic molecules such as CXCL1, CXCL2 and granulocyte colony stimulating factor (GCS-F), which, together with vascular adhesion molecules [24], contribute to neutrophil outflow from blood vessel toward the affected tissue [29]. These data represent the maintenance of a proinflammatory state in these animals that may be related to increased tissue demand. It should also be noted that, although the animals in AI group 
have a stabilized disease, it is possible to visualize a potentially more inflammable tissue than the animals in control group.

The NLR is a widely available and inexpensive laboratory biomarker that quantifies systemic inflammation, being used in human medicine to evaluate prognosis in different types of cancer [8,20]. Recently, its use in inflammatory diseases, such as asthma and atopy, has been highlighted, providing valuable information about disease severity [16,32]. In human atopic dermatitis it has been shown a positive correlation between NLR and lesional score [16], which makes it a potential marker to assess disease activity.

In veterinary medicine, there are few reports of NLR analysis in dogs [27]. In our study, dogs in AII group showed an increased NLR compared to AI and control, which demonstrates the influence of skin injury in systemic parameters. Furthermore, AII group is composed of dogs with greater lesional state, which reflects in higher NLR values. Since this disease is known by its chronicity and may remain stable for years, NLR may be a novel biomarker to evaluate acute exacerbation in CAD and could potentially explain why some patients have longer crisis duration and frequent flares.

In atopic dermatitis, the skin barrier breakdown occurs due to lipoprotein changes in epidermis, which favor the penetration of environmental allergens and commensal microorganisms [30]. These antigens induce a tissue inflammatory response, with the participation of innate lymphoid cells-2 (ILC2) and Th2 cells with consequent production of proinflammatory cytokines such as IL-4, IL-5, IL-10 and IL-13, culminating in IgE synthesis, mast cell degranulation and eosinophil migration to the inflammatory site [35]. This immunological mechanism favors the mobilization of mast cells and eosinophils in groups AI and AII, which can be visualized in histological findings (Figure $1 \mathrm{~F}$ and 1I) [Table 1].

Eosinophils are effector cells of innate and adaptive immunities, exerting their function by activating and releasing substances present in their cytoplasmic granules [3]. Eosinophilic migration to the inflammatory site is mediated by eotaxins, whose synthesis is induced by Th2 cytokines, such as IL-4 and IL-13 [1,31], and is further influenced by Thymic Stromal Lymphopoietin (TSLP), an epithelial cytokine involved in immunopathogenesis of atopic dermatitis [37]. When combined, such cytokines regulate the expression of adhesion molecules, facilitating the arrival of eosinophils at the inflammatory site [36,37]. The presence of these cells favors the maintenance of inflammation in local microenvironment, cellular signaling and consequent tissue destruction $[3,4]$. The persistence of the process affects the skin, resulting in thickening, hydropic degeneration and intercellular edema [23], which were observed in this study (Figure 1C).

The maintenance of the inflammatory state also induces the production of oxidizing substances, which possibly exceed the total antioxidant capacity, generating a situation of oxidative stress [15]. This imbalance can be assessed directly or indirectly by measuring lipid peroxidation products such as MDA and acute phase proteins such as albumin [17]. Serum albumin levels differed between groups, being reduced in AII group, when compared to AI and control. Albumin is a non-enzymatic antioxidant considered to have antiinflammatory and immunomodulatory activities [19]. Therefore, when there is a systemic inflammatory response, there is a reduction in its concentration in order to control the damage by oxidizing substances, as observed in AII.

Among non-enzymatic antioxidants, bilirubin stands out due to its ability to interact and neutralize oxidizing agents, forming hydrophilic substances that will be eliminated by urine [33]. In the present study, it was not possible to observe a difference in serum bilirubin concentration in the evaluated groups. Based on this, it is hypothesized that the consumption of other antioxidants, such as albumin, has priority when compared to bilirubin, and that more intense oxidative processes would be necessary to promote changes in serum bilirubin values, since the animals in the present study had mild lesion grade.

Inflammatory response in CAD includes oxidative stress, which can result in damage to membrane lipids and release of their products $[12,18]$. MDA is reliable and is the most commonly used marker of the overall lipid peroxidation level and the presence of oxidative stress [14], being positively correlated with skin injury in CAD, supporting the hypothesis that increased production of oxidizing agents during the inflammatory process may contribute to tissue damage [18]. In the present study, although an increasing tendency in MDA was showed in AII group, no difference between groups was observed. This result may be 
related with the antioxidant system, such as albumin and bilirubin, which was able to promote an efficient control of oxidant substances.

\section{CONCLUSION}

The presented data demonstrate an increase in systemic and tissue inflammatory response in dogs with atopic dermatitis. The inflammation, however, did not interfere in oxidative balance of the organism in order to cause systemic illness, being necessary studies involving animals with higher lesion grade to evaluate the repercussion of tissue inflammation in homeostasis breakdown. Among the limitations of the study, we highlight the number of dogs, given the difficulty of meeting the inclusion criteria. However, it is noteworthy that this is the first clinical study to evaluate and correlate inflammatory parameters in
CAD according to CADESI, providing relevant data for disease follow-up and monitoring.

\section{MANUFACTURERS}

${ }^{1}$ Cequímica Ltda. Fortaleza, CE, Brazil.

${ }^{2}$ Metaquímica Produtos Ltda. Jaraguá do Sul, SC, Brazil.

${ }^{3}$ GraphPad ${ }^{\circledR}$ Software. San Diego, CA, USA.

Acknowledgements. This work was supported by Coordenação de Aperfeiçoamento de Pessoal de Nível Superior (CAPES) and Financiadora de Estudos e Projetos (FINEP) from Brazil.

Ethical approval. The experimental protocol was approved by the Ethics and Animal Experimentation Committee (EAEC) of Ceara State University under protocol number 9140016/2018. All the owners signed an agreement, authorizing the animals' participation in experiment.

Declaration of interest. The authors declare no conflicts of interest. The authors were responsible for the content and writing of the paper.

\section{REFERENCES}

1 Ahmadi Z., Hassanshahi G., Khorramdelazad H., Zainodini N. \& Koochakzadeh L. 2016. An overlook to the characteristics and roles played by eotaxin net- work in the pathophysiology of food allergies: allergic asthma and atopic dermatitis. Inflammation. 39: 1253-1267.

2 Almela R.M., Rubio C.P., Ceron J.J., Anson A., Tichy A. \& Mayer U. 2018. Selected serum oxidative stress biomarkers in dogs with non-food-induced and food-induced atopic dermatitis. Veterinary Dermatology. 29: 229-e82.

3 Amber K.T., Chernyavsky A., Agnoletti A.F., Cozzani E. \& Grando S.A. 2018. Mechanisms of pathogenic effects of eosinophil cationic protein and eosinophil-derived neurotoxin on human keratinocytes. Experimental Dermatology. 27: 1322-1327.

4 Amber K.T., Valdebran M., Kridin K. \& Grando S.A. 2018. The role of eosinophils in bullous pemphigoid: a developing model of eosinophil pathogenicity in mucocutaneous disease. Frontiers in Medicine. 5: 201.

5 Bertino L., Guarneri F., Cannavò S.F., Casciaro M., Pioggia G. \& Gangemi S. 2020. Oxidative Stress and Atopic Dermatitis. Antioxidants. 9: 196.

6 Chermprapai S., Ederveen T.H.A., Broere F., Broens E.M., Schlotter Y.M., Schalkwijk S.V., Boekhorst J., van Hijum S.A.F.T. \& Rutten V.P.M.G. 2019. The bacterial and fungal microbiome of the skin of healthy dogs and dogs with atopic dermatitis and the impact of topical antimicrobial therapy, an exploratory study. Veterinary Microbiology. 229: 90-99.

7 Cork M.J., Danby S.G., Vasilopoulos Y., Hadgraft J., Lane M.J., Moustafa M., Guy R.H., MacGowan O.L., TaziAhnini R. \& Ward S.J. 2009. Epidermal Barrier Dysfunction in Canine Atopic Dermatitis. Journal of Investigative Dermatology. 129: 1892-1908.

8 Deng Q., He B., Liu X., Yue J., Ying H., Pan Y., Sun H., Chen J., Wang F., Gao T., Zhang L. \& Wang S. 2015. Prognostic value of pre-operative inflammatory response biomarkers in gastric cancer patients and the construction of a predictive model. Journal of Translational Medicine. 13: 66.

9 Draper H.H. \& Hadley M. 1990. Malondialdehyde determination as index of lipid peroxidation. Methods in Enzymology. 186: 421-431.

10 Favrot C., Fischer N., Olivry T., Zwickl L., Audergon S. \& Rostaher A. 2019. Atopic dermatitis in West Highland white terriers - part I: natural history of atopic dermatitis in the first three years of life. Veterinary Dermatology. 31(2): 106-110.

11 Favrot C., Steffan J., Seewald W. \& Picco F. 2010. A prospective study on the clinical features of chronic canine atopic dermatitis and its diagnosis. Veterinary Dermatology. 21: 23-31. 
12 Fuchs J., Zollner T.M., Kaufmann R. \& Podda M. 2001. Redox-modulated pathways in inflammatory skin diseases. Free Radical Biology and Medicine. 30: 337-353.

13 Griffin C.E. \& DeBoer D.J. 2001. The ACVD task force on canine atopic dermatitis (XIV): clinical manifestations of canine atopic dermatitis. Veterinary Immunology and Immunopathology. 81: 255-269.

14 Grotto D., Santa Maria L., Valentini J., Paniz C., Schmitt G., Garcia S.C., Pomblum V.J., Rocha J.B.T. \& Farina M. 2009. Importance of the lipid peroxidation biomarkers and methodological aspects for malondialdehyde quantification. Química Nova. 32: 169-174.

15 Ji H. \& Li X.K. 2016. Oxidative Stress in Atopic Dermatitis. Oxidative Medicine and Cellular Longevity : 1-8.

16 Jiang Y. \& Wencong Ma. 2017. Assessment of Neutrophil-to-Lymphocyte Ratio and Platelet-to-Lymphocyte Ratio in Atopic Dermatitis Patients. Medical Science Monitor. 23: 1340-1346.

17 Kann R.K.C., Seddon J.M., Henning J. \& Meers J. 2012. Acute phase proteins in healthy and sick cats. Research in Veterinary Science. 93(2): 649-654. doi: 10.1016/j.rvsc.2011.11.007.

18 Kapun A.P., Salobir J., Levart A., Kotnik T. \& Svete A.K. 2012. Oxidative Stress Markers in canine atopic dermatitis. Research in Veterinary Science. 92(3): 469-470. doi: 10.1016/j.rvsc.2011.04.014.

19 Kinoshita H., Watanabe K., Azma T., Feng G.G., Akahori T., Hayashi H., Sato M., Fujiwara Y. \& Wakatsuki A. 2017. Human serum albumin and oxidative stress in preeclamptic women and the mechanism of albumin for stress reduction. Heliyon. 3(8): e00369.

20 Krenn-Pilko S., Langsenlehner U., Stojakovic T., Pichler M., Gerger A., Kapp K.S. \& Langsenlehner T. 2016. The elevated preoperative derived neutrophil-to-lymphocyte ratio predicts poor clinical outcome in breast cancer patients. Tumour Biology. 37: 361-368.

21 Li W.C., Mo L.J., Shi X., Lin Z.Y., Li Y.Y., Yang Z., Wu C.L., Li X.H., Qin L.Q. \& Mo W.N. 2018. Antioxidant status of serum bilirubin, uric acid and albumin in pemphigus vulgaris. Clinical and Experimental Dermatology. 43: 158-163.

22 Martins G.D.C., de Oliveira Melo Junior O.A., Botoni L.S., Nogueira M.M., Costa-Val A.P., Blanco B.S., Dutra W.O., Giunchetti R.C., Melo M.M. \& Lemos D.S. 2018. Clinical-Pathological and immunological biomarkers in dogs with atopic dermatitis. Veterinary Immunology and Immunopathology. 205: 58-64.

23 Naidoo K., Jagot F., van den Elsen L., Pellefigues C., Jones A., Luo H., Johnston K., Painter G., Roediger B., Lee J., Weninger W., Gros G.L. \& Forbes-Blom E. 2018. Eosinophils Determine Dermal Thickening and Water Loss in an MC903 Model of Atopic Dermatitis. Journal of Investigative Dermatology. 138: 2606-2616.

24 Oh M.S., Hong J.Y., Kim M.N., Kwak, E.J., Kim S.Y., Kim E.G., Lee K.E., Kim Y.S., Jee H.M., Kim S.H., Sol I.S., Park C.O., Kim W.K. \& Sohn M.H. 2019. Activated Leukocyte Cell Adhesion Molecule Modulates Th2 Immune Response in Atopic Dermatitis. Allergy Asthma and Immunology Research. 11: 677-690.

25 Olivry T., Saridomichelakis M., Nuttall T., Bensignor M., Griffin C.E. \& Hill P.B. 2014. Validation of the Canine Atopic Dermatitis Extent and Severity Index (CADESI)-4, a simplified severity scale for assessing skin lesions of atopic dermatitis in dogs. Veterinary Dermatology. 25: 77-e25.

26 Pierezan F., Olivry T., Paps J.S., Lawhon S.D., Wu J., Steiner J.M., Suchodolski J.S. \& Rodrigues-Hoffmann A. 2016. The skin microbiome in allergen-induced canine atopic dermatitis. Veterinary Dermatology. 27(5): 332-e82.

27 Pierini A., Gori E., Lippi I., Ceccherini G., Lubas G. \& Marchetti V. 2019. Neutrophil-to-lymphocyte ratio, nucleated red blood cells and erythrocyte abnormalities in canine systemic inflammatory response syndrome. Research in Veterinary Science. 126: 150-154. doi: 10.1016/j.rvsc.2019.08.028.

28 Rodrigues-Hoffmann A., Patterson A.P., Diesel A., Lawhon S.D., Ly, H.J., Stephenson C.E., Mansell J., Steiner J.M., Dowd S.E., Olivry T. \& Suchodolski J.S. 2014. The Skin Microbiome in Healthy and Allergic Dogs. Plos One. 9: e83197.

29 Sadik C.D., Kim N.D. \& Luster A.D. 2011. Neutrophils cascading their way to inflammation. Trends in Immunology. 32: 452-460.

30 Santoro D., Marsella R., Pucheu-Haston C.M., Eisenschenk M.N., Nutall T. \& Bizikova P. 2015. Review: pathogenesis of canine atopic dermatitis: skin barrier and host-micro-organism interaction. Veterinary Dermatology. 26: 84-e25.

31 Sastre B., Rodrigo-Muñoz J.M., Garcia-Sanchez D.A., Cañas J.A. \& Del Pozo V. 2018. Eosinophils: old players in a new game. Journal of Investigational Allergology and Clinical Immunology. 28: 289-304. 
32 Shi G., Zhao J. \& Ming L. 2017. Clinical significance of peripheral blood neutrophil-lymphocyte ratio and plateletlymphocyte ratio in patients with asthma. Journal of Southern Medical University. 37(1): 84-88.

33 Shibama S., Ugajin T., Yamaguchi T. \& Yokozeki H. 2019. Bilirubin oxidation derived from oxidative stress is associated with disease severity of atopic dermatitis in adults. Clinical and Experimental Dermatology. 44(2): 153-160.

34 Thijs J.L., Strickland I., Bruijnzeel-Koomen C.A.F.M., Nierkens S., Giovannone B., Knol E.F., Csomor E., Sellman B.R., Mustelin T., Sleeman M.A., Bruin-Weller M.S., Herath A., Drylewicz J., May R.D. \& Hijnen D. 2018. Serum biomarker profiles suggests that atopic dermatitis is a systemic disease. Journal of Allergy and Clinical Immunology. 141(4): 1523-1526.

35 Weidinger S., Beck L.A., Bieber T., Kabashima K. \& Irvine A.D. 2018. Atopic Dermatitis. Nature Reviews Disease Primers. 4: 1-20.

36 Wilson S.R., The L., Batia L.M., Beattie K., Katibah G.E., McClain S.P., Pellegrino M., Estadian D.M. \& Bautista D.M. 2013. The epithelial cell-derived atopic dermatitis cytokine TSLP activates neurons to induce itch. Cell. 155: 285-295.

37 Wong C.K., Hu S., Cheung P.F.Y. \& Lam C.W.K. 2010. Thymic Stromal Lymphopoietin induces chemotactic and prosurvival effects in eosinophils: implications in allergic inflammation. American Journal of Respiratory Cell and Molecular Biology. 43: 305-315. 